\title{
Disrupted architecture of large-scale brain functional connectivity networks in patients with generalized tonic-clonic seizure
}

\author{
Rong Li ${ }^{1}$, Yangyang Yu' ${ }^{1}$ Wei Liao' ${ }^{1}$ Zhiqiang Zhang², Guangming $\mathrm{Lu}^{2}$ and Huafu Chen ${ }^{\text {* }}$
}

*Correspondence:
chenhf@uestc.edu.cn
'The Clinical Hospital
of Chengdu Brain Science
Institute, MOE Key Laboratory
for Neuroinformation
of Ministry of Education,
School of Life Science
and Technology, University
of Electronic Science
and Technology of China,
Chengdu 610054, People's
Republic of China
Full list of author information
is available at the end of the
article

*Correspondence:

chenhf@uestceducn Institute, MOE Key Laboratory for Neuroinformation of Ministry of Education

of Electronic Science and Technology of China, Republic of China

(c) The Author(s) 2017. This article is distributed under the terms of the Creative Commons Attribution 4.0 International License (http://creativecommons.org/licenses/by/4.0/), which permits unrestricted use, distribution, and reproduction in any medium, provided you give appropriate credit to the original author(s) and the source, provide a link to the Creative Commons license, and indicate if changes were made.

\begin{abstract}
Generalized tonic-clonic seizure (GTCS) is characterized by the abnormal functional organization among distant brain regions. Previous studies in GTCS that have comprehensively examined connectivity abnormalities across the complete range of largescale brain networks remain relatively rare. Here, we employed an amount of regions of interest to investigate the intra- and inter-connections among seven large-scale brain networks in GTCS and healthy controls. Network contingency analysis revealed that patients with GTCS exhibit significantly increased connectivity between default mode network (DMN) and frontoparietal network (FPN), between DMN and dorsal attention network, and between somatomotor network and limbic network, and decreased functional connectivity within FPN (all $p$ values were Bonferroni corrected). Consistent with existing evidence, the disrupted functional architecture of the DMN and task-positive network may be related to self-related processes and deficits in cognitive control and attention in patients. These findings support the notion that GTCS is associated with disrupted architecture in large-scale brain networks, providing information for better understanding of the pathophysiological mechanisms of GTCS.
\end{abstract}

Keywords: Brain functional connectivity networks, Resting-state fMRI, Large-scale, GTCS

\section{Introduction}

Idiopathic generalized epilepsy (IGE) is an epileptic syndrome associated with widespread cortical network abnormalities (Blumenfeld et al. 2003). Generalized tonicclonic seizure (GTCS) is the most common phenotype of IGE, and patients with GTCS suffer long-term neuropsychological and cognitive impairments, such as deficits in attention and self-related processing, as well as executive dysfunction (Vlooswijk et al. 2010), which have been demonstrated to be associated with alterations of certain intrinsic functional connectivity networks (Wei et al. 2015). It is increasingly recognized that the human brain is organized into large-scale functional networks (Bressler and Menon 2010; Li et al. 2017). Individual intrinsic functional networks (ICNs) have been implicated in specific neurocognitive functions such as vision, attention, executive function, and self-related processes (Fox et al. 2006; Greicius et al. 2003). Aberrant connectivity 
within and between ICNs has been reported in a number of neurologic and psychiatric conditions (Li et al. 2017; Liao et al. 2010).

Theoretical models of network pathology in GTCS have focused on default mode network (DMN) and its interrelationships with task-positive networks, such as dorsal attention network (DAN) and frontal parietal network (FPN). Intrinsic activity in the DMN provides a baseline state of the human brain associated with spontaneous activities like mind wandering and consciousness maintenance. The suspension of the DMN was assumed to contribute to reduced consciousness during interictal epileptiform discharges in patients with IGE (Gotman et al. 2005). Our previous study has demonstrated lower level of rich club connectivity among DMN regions of the medial prefrontal cortex and the precuneus/posterior cingulate cortex in GTCS (Li et al. 2016). Task-positive networks, including the DAN and the FPN, have been postulated to subserve active cognitive processing (e.g., executive control, attention, and working memory) (Fox et al. 2005). Abnormal functional connectivity within these functional systems has been reported in GTCS (McGill et al. 2012; Tian et al. 2010). Recently, a fMRI study has demonstrated altered functional connectivity among default, attention, and control networks in IGE patients using independent component analysis (ICA) (Wei et al. 2015). However, the ICNs identified using ICA approach so far do not provide a complete description of brain functional architecture as they do not cover the whole cortex (Wei et al. 2015). Other studies have often examined the connectivity of these networks at different individual seed regions making comparisons difficult, and have produced somewhat inconsistent results (Song et al. 2011; Kim et al. 2014; Moeller et al. 2011). Studies in GTCS that have comprehensively examined connectivity abnormalities across the complete range of large-scale brain networks remain relatively rare.

In the current study, we aimed to investigate the potential aberrant intra- and interconnections among large-scale brain functional networks in GTCS. We comprehensively examined functional connectivity disturbances by placing 907 regions of interest (ROIs) at regular intervals throughout the entire brain cortex. A network contingency analysis was employed to identify abnormalities across all seven ICNs and their interconnections in GTCS. Given the growing evidence for altered network organization in GTCS, we hypothesized that patients with GTCS would exhibit altered functional connectivity both within and between specific ICNs such as DMN, attention network, and control network, underlying the cognition dysfunction in patients with GTCS.

\section{Materials and methods}

\section{Subjects}

The datasets in the current study were recruited from Jinling Hospital, Nanjing University School of Medicine, which consist of 55 patients with GTCS (mean age $26.00 \pm 7.94$ ) and 63 age-matched healthy controls (HC) (mean age $25.38 \pm 6.35$ ). Part of these clinical data have been reported in our previous studies (Li et al. 2016; Zhang et al. 2011). Experiments were approved by the Ethics Committee of Jinling Hospital, Nanjing University School of Medicine. All subjects provided written informed consent prior to participation. Based on the International League against Epilepsy criteria (Commission on Classification and Terminology of the International League Against Epilepsy 1989), all patients match the following criteria: (1) clinical features of GTCS were detected, such 
as twitching limbs, out of consciousness, and generalized seizures; (2) generalized polyspike waves were observed in their scalp EEG; (3) no psychiatric or neurological etiology history; (4) no focal lesions in anatomical MRI, and 5) right handedness. In addition, $\mathrm{HC}$ had no current or lifetime neurologic disorders or psychiatric illnesses and no gross abnormalities on brain MRI.

\section{Data acquisition}

Imaging was acquired on a 3T MR scanner (TIM Trio; Siemens Medical Solutions, Erlangen, Germany) with a standard birdcage head transmit and receive coil at Jinling Hospital, Nanjing, China. Data of patients were acquired during the interictal periods. Foam padding was used to minimize head motion for all participants. Functional images were acquired using a single-shot, gradient-recalled echo-planar imaging sequence $\left(\mathrm{TR}=2000 \mathrm{~ms}, \mathrm{TE}=30 \mathrm{~ms}\right.$, and $\left.\mathrm{FA}=90^{\circ}\right)$. Thirty transverse sections $\left(F O V=240 \times 240 \mathrm{~mm}^{2}\right.$, in-plane matrix $=64 \times 64$, slice thickness $=4 \mathrm{~mm}$, interslice gap $=0.4 \mathrm{~mm}$, voxel size $=3.75 \times 3.75 \times 4 \mathrm{~mm}^{3}$ ), aligned along the anterior commissure-posterior commissure line, were acquired. For each subject, a total of 250 volumes were acquired, resulting in a total scan time of $500 \mathrm{~s}$. The subjects were told to relax, hold still, keep their eyes closed without falling asleep, and think of nothing in particular.

\section{Preprocessing}

Functional images were preprocessed using Data Processing Assistant for Resting-State fMRI software (DPARSF, Advanced Edition, V4.0) (http://www.restfmri.net/forum/). For each subject, the initial 10 functional images were discarded to ensure steady-state longitudinal magnetization. Subsequently, we performed slice timing and realignment for the remaining 240 images to correct for the acquisition delay between slices and the head motion. Then, the corrected images were normalized to the standard SPM8 echoplanar imaging template, resampling to $3 \times 3 \times 3 \mathrm{~mm}^{3}$. Nuisance signals (i.e., 24 head motion parameters, averaged signals from CSF, white matter) were regressed out using multiple linear regression analysis. Finally, the resulting images were detrended to move linear trends and temporal filtered with a bandpass filter $(0.01-0.08 \mathrm{~Hz})$. We required that the transient movement during scanning was no more than $3 \mathrm{~mm}$ of translation and $3^{\circ}$ of rotation. Since some recent studies have shown that functional connectivity analysis is sensitive to gross head motion effects (Power et al. 2012; Van Dijk et al. 2012), the mean framewise displacement (FD) was also calculated to further determine the comparability of head movement across groups. The FD threshold for excessive motion was set at $0.3 \mathrm{~mm}$.

\section{Functional network construction}

We placed regions of interest (ROIs) with nineteen voxels at $12-\mathrm{mm}$ intervals across the whole brain to generate the whole brain functional connectome for each subject. Given that the aim of this study is to investigate the intra- and inter-connections among largescale networks in GTCS and HC, we employed the ICN parcellation template from Yeo et al. (2011), which parcellated the cortical brain into seven cortical networks (including visual network (VN), somatomotor network (SMN), dorsal attention network (DAN), ventral attention network (VAN), limbic network (LN), frontoparietal network (FPN), 
and default modal network (DMN)). Since our main interest was cortical ICNs, we removed all ROIs that fell more than $5 \mathrm{~mm}$ from the ICN parcellation of the brain (Yeo et al. 2011), yielding 907 ROIs in total. Using the preprocessed resting-state fMRI time series, we extracted spatially averaged time series from each of the ROIs. Then, Pearson's correlation coefficients were calculated pairwise between time courses for each of the 907 ROIs, producing a temporal correlation matrix $(N \times N$, where $N=907$ is the number of regions of interest) for each subject. Those correlation matrices were then normalized by Fisher's $\mathrm{r}-\mathrm{z}$ transformation to improve the normality.

\section{Network contingency analysis}

To address the question of whether and where the GTCS group showed significantly different intra- and inter-connections among large-scale functional networks, we conducted the network contingency analysis. This analysis takes a population-based approach to the question of when two networks exhibit disrupted connectivity. Network contingency analysis is composed of the following three steps.

Firstly, the multiple regression analysis was employed for each edge to model the effect of disease (GTCS versus $\mathrm{HC}$ ), while controlling the effect of nuisance variables including age, gender, and head movement characterized by the framewise displacement (Power et al. 2012). The modal would generate a statistical $T$ value for each edge, indicating to what extent the GTCS group is different from the HC. We then thresholded delta connectome based on statistical significance at $p<0.005$. Those edges surviving the threshold would be used for further analysis.

Secondly, we reorganized those suprathreshold edges based on network affiliation. Briefly, we used the network map of Yeo et al. (2011), which parcellated the brain into seven major networks. Those networks were employed to generate the cross-tabulation map with 28 nonredundant cells. Each cell represents the number of edges linking pairs of networks.

Thirdly, to test the hypothesis of whether the GTCS group showed significant difference in functional connectivity within/between networks compared with the HC group, the nonparametric permutation approach (5000 permutations) was employed. For each permutation, each participant was randomly assigned to one of the two groups with the same size as the original group of GTCS and HC. Multiple regression analysis was performed and the statistical $T$ value for each edge was obtained. The number of suprathreshold edges was then calculated for each pair of networks. The whole procedure would result in a null distribution of the number of suprathreshold edges for each cell. A $p$ value was assigned to each cell by computing the proportion of the number of suprathreshold edges exceeding the null distribution values. We performed the contingency analysis for each cell and corrected for multiple comparisons using Bonferroni correction with $p<0.05$.

We were also interested in the directionality of changes. Cells change in positive direction if the number of positive suprathreshold edges is larger than the number of negative suprathreshold edges, and vice versa. We generated 3-dimensional visualization of cells showing significant changes in functional connectivity between the GTCS group and the HC group, by employing BrainNet Viewer (Xia et al. 2013). 
Furthermore, Shepherd's pi correlation analysis was performed to explore the potential relationship between abnormal functional connectivity and disease characteristics (disease duration and seizure frequency). The statistical significance level for the correlation analysis was set at $p<0.05$ (uncorrected).

\section{Results}

\section{Altered intra- and inter-connections among functional connectivity networks}

Network contingency analysis revealed statistically significant effects in four cells (where each cell represents the set of connections between two ICNs). Compared with HC, GTCS showed significantly altered functional connectivity within FPN $(p=0.0004$,$) and altered connectivity between DMN and FPN (p=0.0010)$, between DMN and DAN $(p=0.0006)$, and between SMN and LN ( $p=0.0004)$, where all $p$ values were Bonferroni corrected (Fig. 1). Three pairs of networks are shaded in warm color, suggesting that there was a preponderance of connections showing a significant increase in GTCS compared to HC. Within FPN there was a preponderance of connections showing significantly decreased connectivity, which are shaded in cool color. Those full network interrelationships were visualized on sagittal and axial views in Fig. 2.

\section{Clinical correlation analysis}

Shepherd's pi correlation analysis showed that the disrupted functional connectivity strength within FPN was negatively correlated with the disease duration in GTCS patients (Fig. 3; $r=-0.35, p=0.0230$ ). There was no significant correlation between connectivity of other pairs of networks and disease characteristics.

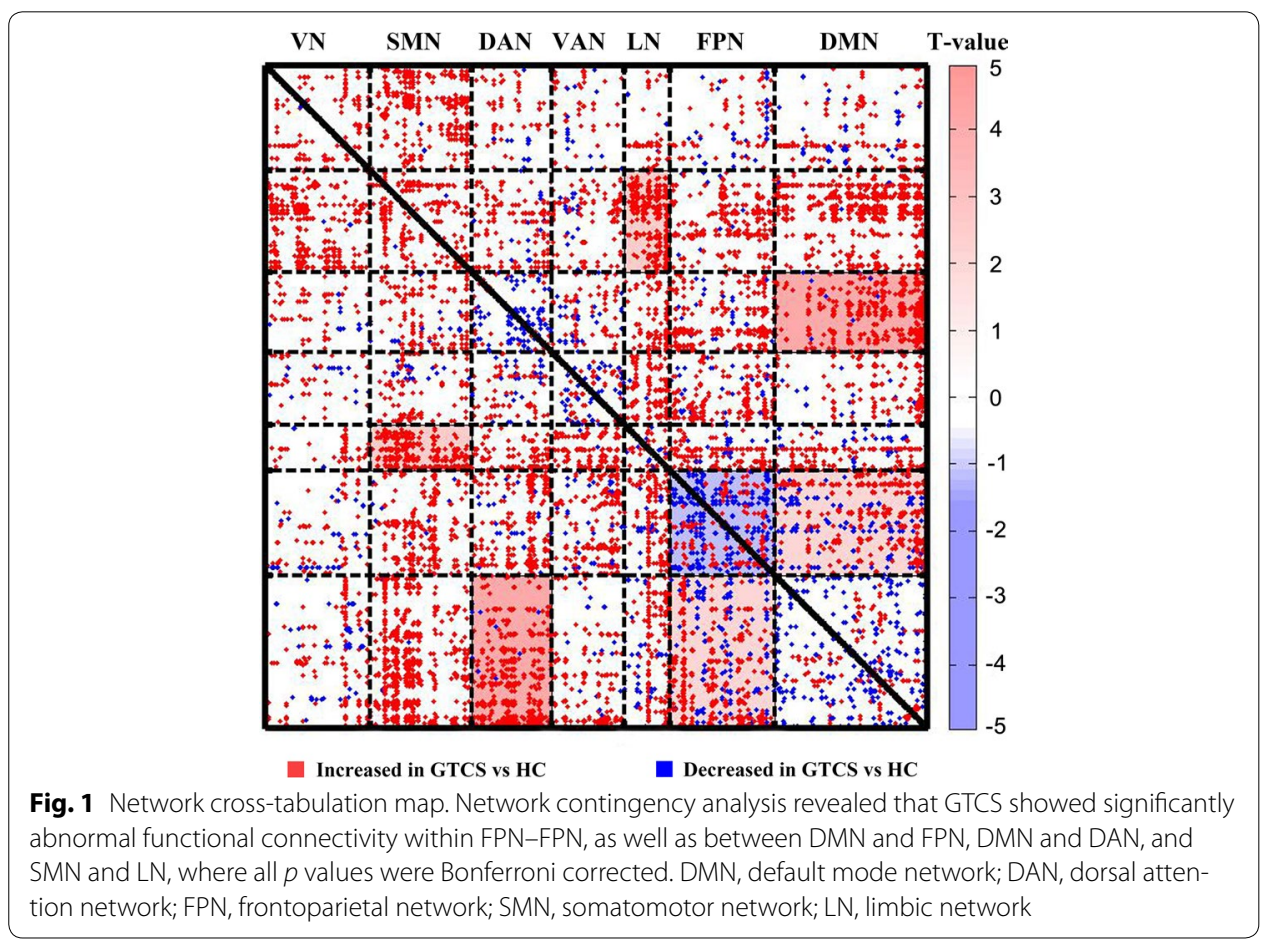



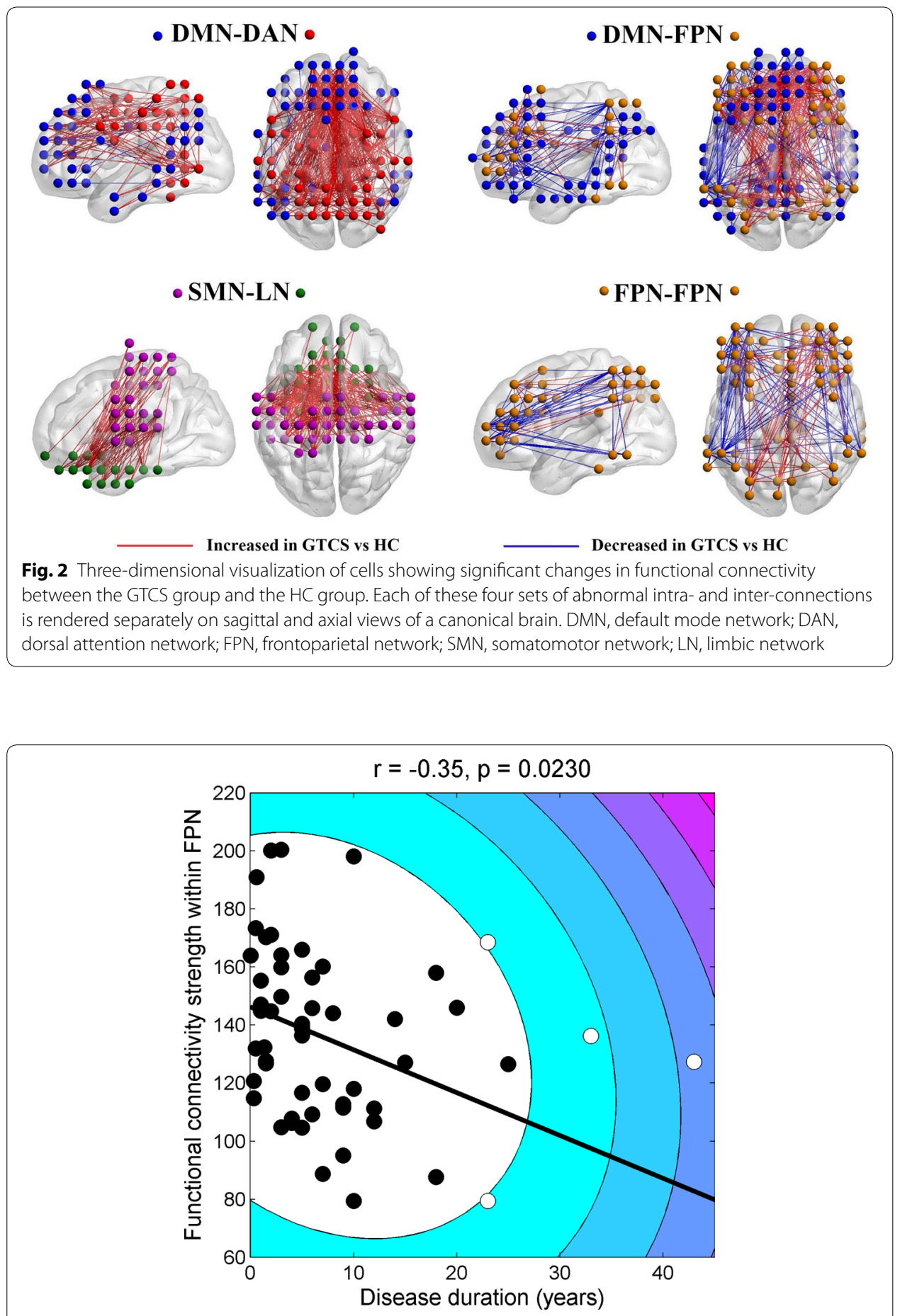

Fig. 3 Correlation between the functional connectivity strength within FPN and the disease duration in GTCS patients. The decreased functional connectivity strength within FPN was significantly negatively correlated with longer disease duration (Shepherd's pi correlation: $r=0.35, p=0.0230$ ). FPN, frontoparietal network

\section{Discussion}

The present study investigated intra- and inter-connections among large-scale brain 
intrinsic functional networks in patients with GTCS applying network contingency analysis. Consistent with previous studies (Wei et al. 2015; Zhang et al. 2011), we demonstrated that GTCS exhibits altered connectivity between default network, which is involved in self-related processes, and FPN and DAN, which is involved in cognitive control and attention-demanding cognition functions, respectively. We also found that FPN exhibited distributed alterations within intra-connections, and this decreased connectivity pattern was negatively correlated with longer epilepsy duration. These findings add to the growing evidence that GTCS is likely to be associated with functional network connectivity abnormalities.

An important finding of the current study is the significant increased inter-connections between default network and task-positive networks, including the DAN and the FPN. In the prior studies (Fox et al. 2005), the relationship between the default network and task-positive network was referred to as "anticorrelated," suggesting a discriminate role that segregates neuronal processes contributing to opposite goals or competing representations. The default network is involved in introspective orientation of attention during activities such as autobiographical memory, prospective thought, and self-related processing. Task-positive networks, in contrast, are commonly activated with a variety of cognitive control processes, particularly those involving conflict monitoring, information integration, and response selection. DAN and FPN are key task-positive networks that perceive externally directed stimuli and regulate switching between default network and task-positive modes of cognition. Previous studies have demonstrated that IGEGTCS is likely to be associated with a disrupted brain organization probably derived from abnormal functional interactions among default, attention, and control networks (Wei et al. 2015; McGill et al. 2012; Wang et al. 2011). Consistent with previous findings, the current study confirmed that the normative pattern of anticorrelation was disrupted with increased positive connectivity between the DMN and the DAN and the FPN in patients with GTCS. This altered connectivity pattern could be an indication as dysfunction in the shifting balance between these intrinsic functional networks due to epileptic activity, perhaps associated with deficits in cognitive control and attention.

Consistent with previous network studies on epilepsy (Wei et al. 2015), our results also showed significantly decreased functional connectivity within FPN in GTCS. Furthermore, this decreased connectivity pattern was negatively correlated with longer epilepsy duration $(r=0.35, p=0.0230)$. The regions within FPN are activated during tasks demanding cognitive control and executive function (Spreng et al. 2013). Patients with GTCS have been associated with impairments in executive function (Hommet et al. 2006). Accordingly, the disrupted functional interactions among regions within FPN may reflect the aberrant functional network segregation in GTCS, as a result of deficits in goal-directed cognitive processes.

\section{Conclusions}

In sum, by applying large-scale network analysis approach, we have demonstrated the abnormal inter-connections between default network and task-positive networks and the disrupted intrarelationships within the FPN control network. The disrupted functional architecture of the DMN and the task-positive network may be related to selfrelated processes and deficits in cognitive function and attention in patients. Our 
findings support the notion that GTCS is associated with disrupted architecture in large-scale brain networks, providing information for better understanding of the pathophysiological mechanisms of GTCS.

\begin{abstract}
Abbreviations
GTCS: generalized tonic-clonic seizure; ICNs: intrinsic functional networks; DAN: dorsal attention network; DMN: default mode network; FPN: frontoparietal network; LN: limbic network; SMN: somatomotor network; VAN: ventral attention network; VN: visual network.
\end{abstract}

\title{
Authors' contributions
}

RL and HFC conceived and designed the study. ZQZ and GML collected the clinical data. RL, WL, and YYY analyzed the data and also wrote the paper. All authors read and approved the final manuscript.

\section{Author details}

${ }^{1}$ The Clinical Hospital of Chengdu Brain Science Institute, MOE Key Laboratory for Neuroinformation of Ministry of Education, School of Life Science and Technology, University of Electronic Science and Technology of China, Chengdu 610054, People's Republic of China. ${ }^{2}$ Department of Medical Imaging, Jinling Hospital, Nanjing University School of Medicine, Nanjing 210002, China.

\section{Acknowledgements}

The authors thank the patients and volunteers for participating in this study.

\section{Competing interests}

All authors declare that they have no competing interests.

\section{Ethics approval and consent to participate}

Not applicable.

\section{Funding}

This work was supported by the 863 project (2015AA020505), the National Natural Science Foundation of China (61533006 and 81471653), and the China Postdoctoral Science Foundation (2013M532229).

\section{Publisher's Note}

Springer Nature remains neutral with regard to jurisdictional claims in published maps and institutional affiliations.

Received: 26 October 2017 Accepted: 23 November 2017

Published online: 29 November 2017

\section{References}

Blumenfeld $\mathrm{H}$ et al (2003) Selective frontal, parietal, and temporal networks in generalized seizures. Neuroimage 19(4):1556-1566

Bressler SL, Menon V (2010) Large-scale brain networks in cognition: emerging methods and principles. Trends Cogn Sci 14(6):277-290

Commission on Classification and Terminology of the International League Against Epilepsy (1989) Proposal for revised classification of epilepsies and epileptic syndromes, vol 30. Commission on Classification and Terminology of the International League Against Epilepsy, Epilepsia, pp 389-399

Fox MD et al (2005) The human brain is intrinsically organized into dynamic, anticorrelated functional networks. Proc Natl Acad Sci USA 102(27):9673-9678

Fox MD et al (2006) Spontaneous neuronal activity distinguishes human dorsal and ventral attention systems. Proc Natl Acad Sci USA 103(26):10046-10051

Gotman J et al (2005) Generalized epileptic discharges show thalamocortical activation and suspension of the default state of the brain. Proc Natl Acad Sci USA 102(42):15236-15240

Greicius MD et al (2003) Functional connectivity in the resting brain: a network analysis of the default mode hypothesis. Proc Natl Acad Sci USA 100(1):253-258

Hommet C et al (2006) Idiopathic epileptic syndromes and cognition. Neurosci Biobehav Rev 30(1):85-96

Kim JB et al (2014) Altered thalamocortical functional connectivity in idiopathic generalized epilepsy. Epilepsia 55(4):592-600

Li R et al (2016) Disrupted structural and functional rich club organization of the brain connectome in patients with generalized tonic-clonic seizure. Hum Brain Mapp 37(12):4487-4499

Li R et al (2017) Epileptic discharge related functional connectivity within and between networks in benign epilepsy with centrotemporal spikes. Int J Neural Syst 27(7):1750018

Liao W et al (2010) Selective aberrant functional connectivity of resting state networks in social anxiety disorder. Neuroimage 52(4):1549-1558

McGill ML et al (2012) Default mode network abnormalities in idiopathic generalized epilepsy. Epilepsy Behav 23(3):353-359

Moeller F et al (2011) Functional connectivity in patients with idiopathic generalized epilepsy. Epilepsia 52(3):515-522 
Power JD et al (2012) Spurious but systematic correlations in functional connectivity MRI networks arise from subject motion. Neuroimage 59(3):2142-2154

Song M et al (2011) Impaired resting-state functional integrations within default mode network of generalized tonicclonic seizures epilepsy. PLoS ONE 6(2):e17294

Spreng RN et al (2013) Intrinsic architecture underlying the relations among the default, dorsal attention, and frontoparietal control networks of the human brain. J Cogn Neurosci 25(1):74-86

Tian Y et al (2010) Attention networks in children with idiopathic generalized epilepsy. Epilepsy Behav 19(3):513-517

Van Dijk KR, Sabuncu MR, Buckner RL (2012) The influence of head motion on intrinsic functional connectivity MRI. Neuroimage 59(1):431-438

Vlooswijk MC et al (2010) Functional MRl in chronic epilepsy: associations with cognitive impairment. Lancet Neurol 9(10):1018-1027

Wang Z et al (2011) Altered resting state networks in epileptic patients with generalized tonic-clonic seizures. Brain Res 1374:134-141

Wei HL et al (2015) Altered functional connectivity among default, attention, and control networks in idiopathic generalized epilepsy. Epilepsy Behav 46:118-125

Xia M, Wang J, He Y (2013) BrainNet Viewer: a network visualization tool for human brain connectomics. PLoS ONE 8(7):e68910

Yeo BT et al (2011) The organization of the human cerebral cortex estimated by intrinsic functional connectivity. J Neurophysiol 106(3):1125-1165

Zhang Z et al (2011) Altered functional-structural coupling of large-scale brain networks in idiopathic generalized epilepsy. Brain 134(10):2912-2928

\section{Submit your manuscript to a SpringerOpen ${ }^{\circ}$ journal and benefit from:}

- Convenient online submission

- Rigorous peer review

- Open access: articles freely available online

- High visibility within the field

- Retaining the copyright to your article

Submit your next manuscript at $\boldsymbol{\nabla}$ springeropen.com 